\section{Beautifully accurate impressions}

\author{
AFFINIS from COLTENE allows \\ clinicians to create beautifully accurate \\ impressions. \\ The benefits of AFFINIS include: \\ excellent flow properties even in moist \\ environments, excellent readability, an \\ ideal working time and over $98 \%$ perfect \\ reproduction of marginal contours. \\ Also, AFFINIS impression material is \\ fully autoclavable, without the loss of its \\ various properties. \\ Part of the premium AFFINIS range
}

is BLACK EDITION, a tray material that reaches with gold or silver-coloured AFFINIS PRECIOUS. The unique pigmentation vastly improves visuals by reducing light diffusion, allowing the lab to create well-fitting impressions the first time around.

AFFINIS has pushed boundaries - add it to your restorative materials today.

To find out more visit www.coltene.com, email info.uk@coltene.com or call 01444 235486.

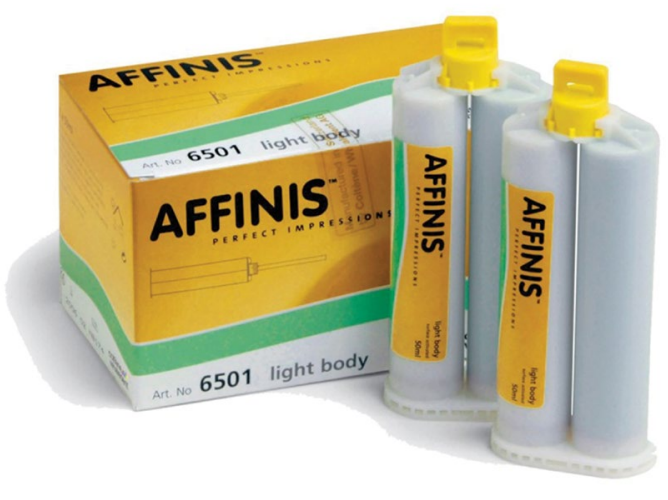

The 13 titanium-reinforced membrane options are easy to trim and versatile in shaping and positioning.

creos syntoprotect is now available in Europe and the United States, where the line of creos regenerative products is conveniently available from the Nobel Biocare online store or through any Nobel Biocare Sales Representative.

creos syntoprotect will become available in additional geographies in the coming months.

For more information on creos and the new creos syntoprotect, dental professionals can visit nobelbiocare.com/creos or contact their local Sales Representative.

\title{
Activation in every aligner stage
}

Align Technology, Inc. recently announced the commercial availability of Invisalign G8 with SmartForce Aligner Activation, the company's latest innovations. Invisalign G8 is informed by the company's foundational biomechanics for clear aligners, with a database of more than 9 million Invisalign patients to optimise tooth movements and further improve predictability for frequently treated cases of crowding, crossbite, and deep bite. The company previously announced that Invisalign G8 would be available in the first quarter of 2021.

With SmartForce Aligner Activation, selected areas of the aligner surface are specifically contoured to apply optimal forces to the tooth surfaces in order to control the location, direction and intensity of the force and produce the desired outcome, whilst minimising unwanted movement. Specific, strategic contact areas between the aligner and the tooth are created by SmartForce Aligner Activation and work in concert with SmartForce features for even greater control of the force systems.

Data demonstrate that Precision Bite Ramps improve intrusion in deep bite cases, so in addition, with Invisalign G8, doctors can now select automatic placement of Precision Bite Ramps during the prescription process.

To summarise the new G8 features: For crowding and crossbite cases: Smart Force Aligner Activation aids in posterior arch expansion by working synergistically with New Optimised Expansion Support attachments or Optimised Expansion Support and Rotation attachments to reduce the potential for buccal crown tipping during posterior arch expansion.

For deep bite cases: SmartForce Aligner Activation supports anterior intrusion with improvements in the treatment plan set-ups to level the curve of Spee and demonstrates up to $2 \mathrm{x}$ improvement in predictability of anterior intrusion.

Invisalign G8 with new SmartForce Aligner Activation ensures sufficient and consistent activation in every aligner stage to help doctors get more of the desired movements from every aligner in the treatment of crowding, crossbite, and deep bite.

For more information visit www. invisalign.com/G8 